\title{
Self-oriented $\mathrm{CoFe}_{2} \mathrm{O}_{4}$ composites for non-reciprocal microwave components
}

\author{
A. Tchangoulian ${ }^{1,3, \text { a }}$, E. Abou Diwan ${ }^{1}$, D. Vincent ${ }^{1}$, S. Neveu ${ }^{2}$, C. Nader ${ }^{3}$ and R. Habchy ${ }^{3}$ \\ ${ }^{1}$ LT2C Laboratory, Jean-Monnet University, 25 rue Dr. Rémy Annino F-42000 Saint-Etienne, France \\ ${ }^{2}$ UPMC Univ Paris 06, UMR 7195, PECSA, F-75005, Paris, France \\ ${ }^{3}$ Research Platform in Nano Sciences and Nanotechnologies, Campus Fanar, Lebanese University, 90239, Jdeidet, \\ Lebanon
}

\begin{abstract}
In telecommunication systems, heavy bulky magnets are used to establish the proper functioning of a circulator by ensuring the uniform orientation of the ferrite material's magnetic moment.

Thus to develop an unbiased coplanar microwave circulator, the approach based on "ferromagnetic nanowired composite substrates" was promising. The idea was to do a magnetophoretic deposition of nanocoloidal cobalt ferrite nanoparticles into porous alumina membranes and permanently orient them uniformly. Therefore, in order to check the orientation possibility of the nanoparticle, samples of magnetic thin films on glass substrates were synthetized from $\mathrm{CoFe}_{2} \mathrm{O}_{4}$ nanoparticles dispersed in a silica sol-gel matrix using the dip-coating technique with and without a uniformly applied magnetic field. To investigate the magnetic behavior of the prepared samples, the Faraday rotation as a function of the applied magnetic field was measured using a spectral polarimeter. The unambiguous qualitative difference between the Faraday rotation hysteresis loops shows a large variation of coercive $\left(\mu_{0} \mathrm{H}_{c}\right)$ and remanent field $\left(\frac{M_{r}}{M_{s}}\right)$ values, thus proving the orientation of the nanoparticles.

Such nanocomposite is a promising candidate for future miniature microwave circulators fabrication.
\end{abstract}

\section{Introduction}

Microwave circulators have had a serious evolution in the last few decades, whether in performance or in miniaturization, yet the latter is limited due to the need of bulky magnets to orient the ferrite crystal and assure the function of circulation [1]. There are many studies in the world that deal with this issue and many approaches to these studies such as circulators using hexaferrite [2] or ferromagnetic nanowires [3-6]. The idea was to create a circulator based on ferromagnetic nanowired composite substrates, while removing the bulky magnets, using cobalt ferrite nanoparticles deposed into porous alumina membranes. Thus by using a self-biased ferrite nanocomposite, based on ferrite nanoparticles in a dielectric host matrix and controlling its concentration, one can control the main physical parameters needed to determine the performance of the ferrite crystal, namely, the saturation magnetization $\mathrm{M}_{\mathrm{s}}$, the damping factor $\alpha$ and more...[7] . And the choice of the dielectric material was based on the fact that a low dielectric loss material was necessary to reduce the insertion losses.
In this paper, the possibility of $\mathrm{CoFe}_{2} \mathrm{O}_{4}$ nanoparticles orientation in a sol gel matrix was tested before even filling a porous alumina with nanoparticles, to assure the total evaporation of all solvents and the orientation of the nanoparticles in the silica. Various samples were prepared, using the dip coating method to depose a ferrofluid solution mixed with a silica sol-gel matrix on glass substrates, under a DC magnetic field. A spectral polarimeter was used to observe the Faraday rotation produced by the material and comparing the remanent and saturation fields of a perfectly oriented magnetic material to a non-oriented magnetic material. After the success of these investigations to verify the possibility of $\mathrm{CoFe}_{2} \mathrm{O}_{4}$ nanoparticles' orientation, deposition of the nanoparticles in the porous alumina was performedin order to achieve an oriented ferromagnetic nanoparticle self-biased substrate.

\footnotetext{
ardaches Tchangoulian: ardo_s_cabana@hotmail.com
} 


\section{Numerical study of a coplanar circulator}

At first, it was necessary to simulate a circulator while replacing the regular ferrite crystal with an oriented magnetic composite material, namely a porous alumina material filled with oriented nanoparticles in a silica matrix. But due to the model complications and hardware limitations we decided to use an effective material that embodies the magnetic functions of the latter.

\subsection{Structure and geometric characteristics}

A coplanar circulator consists of a main central conductor with three ports and a ground planes placed on the same level. Another ground plane placed on an inferior level and in between these two levels is the ferrite crystal or in our case the composite material surrounded with a dielectric materialas shown in figure 1.

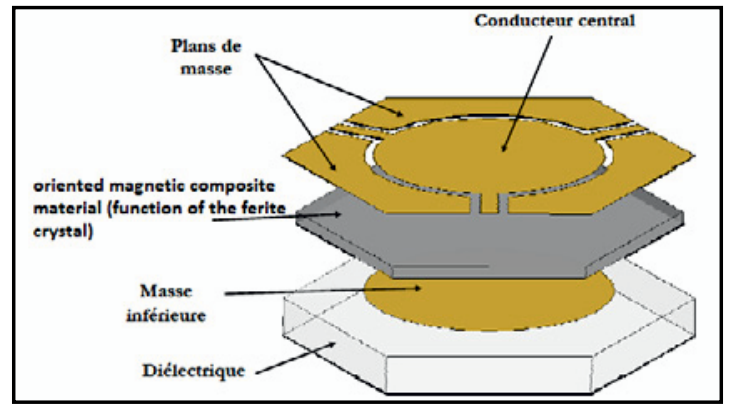

Fig. 1.Structure of the designed circulator with an effective composite material replacing the ferrite crystal.

The dimensions of the circulator were chosen according to a previously owned mask in the lab [2] that has shown successful results. Having a width of $72 \mu \mathrm{m}$ for the transmission lines separated by a distance of $40 \mu \mathrm{m}$ from the coplanar ground. The radius of the central disc was $1 \mathrm{~mm}$ and that of the inferior ground plane is of $1.04 \mathrm{~mm}[2]$.

The composite material simulated has a thickness of $180 \mu \mathrm{m}$ presenting the case of three filled porous membranes because a single membrane has a thickness of $60 \mu \mathrm{m}$. As was said before, to simulate the device using HFSS, the $\mathrm{CoFe}_{2} \mathrm{O}_{4}$ particles and the silica nanowires in the porous membrane are considered as a saturated effective medium with an effective permittivity [1]:

$$
\begin{gathered}
\varepsilon_{\mathrm{eff}}=(1-\mathrm{P}) \varepsilon_{\mathrm{alu}}+\mathrm{P}\left(\beta \varepsilon_{\mathrm{comp}}+(1-\beta) \varepsilon_{\mathrm{air}}\right) \\
\varepsilon_{\mathrm{comp}}=\phi \varepsilon_{\mathrm{fer}}+(1-\phi) \varepsilon_{\text {silica }}
\end{gathered}
$$

where $\mathrm{P}=50 \%$ is the porosity of the alumina membrane, $\beta=80 \sim 100 \%$ is the filling ratio of the membrane, $\phi$ is the volume fraction of $\mathrm{CoFe}_{2} \mathrm{O}_{4}$ in the silica matrix , $\varepsilon_{\text {alu }} \approx 10$ is the permittivity of alumina, $\varepsilon_{\text {air }} \approx 1$ is the permittivity of air, $\varepsilon_{\mathrm{fer}} \approx 13$ is the permittivity of cobalt ferrite and $\varepsilon_{\text {silica }} \approx 11.7$ is permittivity of silica. The effective permittivity can be controlled by the filling ratio, thus we find $\varepsilon_{\mathrm{eff}} \approx 10$ for $\beta=80 \%$ and $\varepsilon_{\mathrm{eff}} \approx 11.4$ for $\beta=100 \%$ and $\phi=80 \%$. The saturation magnetization $\mathrm{M}_{\mathrm{s}}$ of cobalt ferrite nanoparticles is of $0.53 \mathrm{~T}$ and that of the composite effective material depends on the percentage of nanoparticle in the substrate and the filling ratio based on the equation 3;

$$
\mathrm{M}_{\text {scomp }}=\mathrm{P} \beta \phi \mathrm{M}_{\mathrm{s}}
$$

Thus having $\mathrm{M}_{\text {scomp }} \approx 0.1325 \mathrm{~T}, \Delta \mathrm{H}=2273.6 \mathrm{~A} / \mathrm{m}$, the damping factor $\alpha \approx 10^{-3}$ and a relative permittivity of 10.8 for a $\phi=50 \%$ and $\beta=100 \%$, the circulator was simulated.

\subsection{Simulation results and discussion}

The circulator was simulated on the Ansoft HFSS software. It is a finite element 3D full electromagnetic simulating tool essential for high frequency and high speed components.

Figure 2 presents the three S parameters: S11 for reflection, S21 for transmission and $\mathrm{S} 31$ for isolation. As can be seen the circulators functions in the frequency band of 37 to $41 \mathrm{GHz}$, the circulation occursat $38.9 \mathrm{GHz}$ having $-27 \mathrm{~dB}$ of reflection, $-17 \mathrm{~dB}$ isolation and $-1.51 \mathrm{~dB}$ transmission loss that is due to dielectric losses.

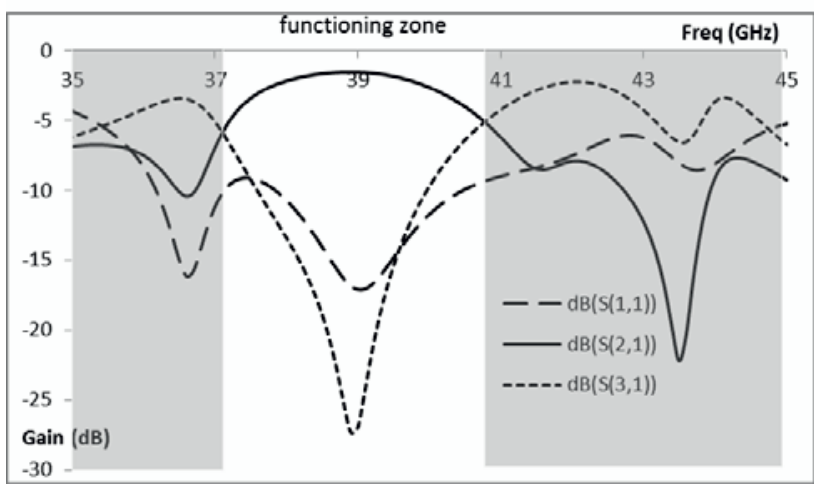

Fig. 2.S parameters of the simulated circulator (HFSS results)

These results are promising but before beginning the circulator fabrication it is important to verify the possibility of particle orientation in the silica matrix and that they will not return to their initial states in the normal conditions.

\section{Experimental details}

\subsection{The ferrofluide mixed with TEOS}

\subsection{1 $\mathrm{CoFe}_{2} \mathrm{O}_{4}$ Nanoparticle preparation}

Spherical $\mathrm{CoFe}_{2} \mathrm{O}_{4}$ nanoparticles weresynthetized by coprecipitation of $\mathrm{Co}(\mathrm{II})$ and $\mathrm{Fe}(\mathrm{III})$ hydroxides followed by a hydrothermal decomposition treatment at $200^{\circ} \mathrm{C}$ for 24 hours. The average nanoparticle diameter is between 15 and $18 \mathrm{~nm}$ as shown on figure 3 . These particles were 
prepared in PECSA Laboratory in Paris and were sent to us to conduct our tests.
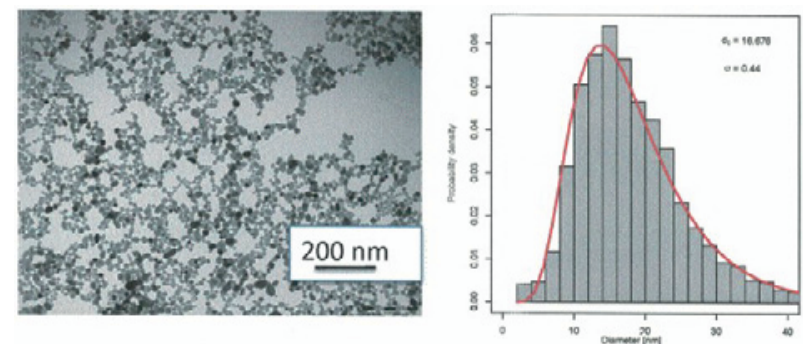

Fig. 3. TEM image of $\mathrm{CoFe}_{2} \mathrm{O}_{4}$ hydrothermal treated nanoparticles and corresponding histogram.

\subsubsection{TEOS preparation}

The TEOS solution is prepared by mixing the Tetraethyl orthosilicate (TEOS) with Ethanol (98\% alcohol concentration) and HCL $(0.1 \mathrm{~mol} / \mathrm{L})$ [9]. The proportions of each ingredient depend on their number of mole as is shown in the equation 4 [9];

$$
\frac{\mathrm{m}_{\mathrm{TEOS}}}{\mathrm{m}_{\mathrm{EtOH}}}=0.5, \frac{\mathrm{m}_{\mathrm{H}_{2} \mathrm{O}}}{\mathrm{m}_{\mathrm{TEOS}}}=2, \frac{\mathrm{m}_{\mathrm{TEOS}}}{\mathrm{m}_{\mathrm{HCl}}}=0.01
$$

Where $\mathrm{m}_{\mathrm{TEOS}}, \mathrm{m}_{\mathrm{EtOH}}, \mathrm{m}_{\mathrm{H}_{2} \mathrm{O}}$ and $\mathrm{m}_{\mathrm{HCL}}$ are respectively the number of mole of TEOS, EtOH, $\mathrm{H}_{2} \mathrm{O}$ and $\mathrm{HCl}$. The TEOS final solution was prepared using $61.5 \%$ tetraethyl orthosilicate mixed with $28.5 \%$ ethanol. After rigorous agitation for a few minutes, $10 \% \mathrm{HCl}$ was added and the solution was agitated slowly for several hours.

\subsubsection{Sol gel process}

A process is called sol-gel when a solution has a solgel transition where the former becomes a rigid mass [8], in this paper, it's the polymerization of the TEOS mixed with water and ethanol after the addition of the acid (catalyst). The chemical steps of sol-gel polymerization are hydrolysis and condensation, these steps result a chemical Si-O-Si linkage to create the silica sol-gel that is processed by drying to evaporate the water and ethanol and therefore having a solid state silica $[5,6]$.

\subsection{Sample preparation}

To verify the orientation of cobalt ferrite particles and the possibility to disperse them in a certain volume of silica while keeping their orientation, at first various samples on glass substrates were made.To compare the case of nonoriented nanoparticles with oriented ones, for each prepared solution of nanoparticles and TEOS there were two samples made: one dip coated and left to dry without a magnetic field and the other one, dip coated and left to dry under a magnetic field of $0.5 \mathrm{~T}$ for at least one hour to allow the ethanol and water to evaporate completely, figure 4.During this time the volume of the gel is reduced approximately by $80 \%$ as a result a silica xerogel [8] that will hold the nanoparticles stillis created, preventing any attempt to return to the neutral state.

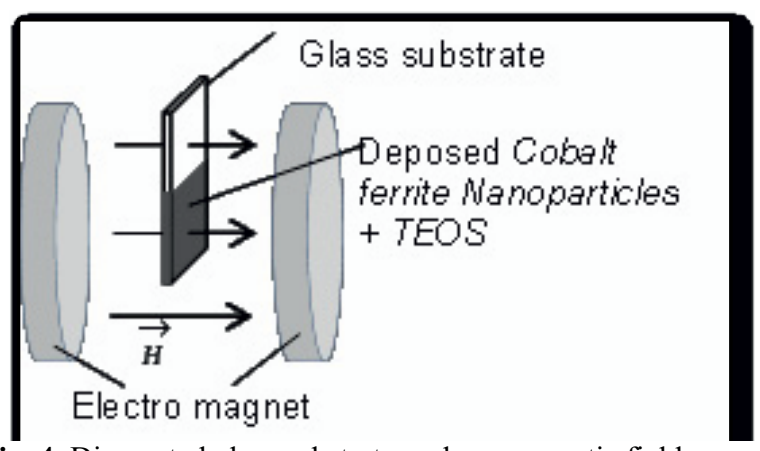

Fig. 4. Dip coated glass substrate under a magnetic field.

The final solution was prepared mixing $50 \%$ of the prepared TEOS with $50 \%$ of $\mathrm{CoFe}_{2} \mathrm{O}_{4}$ ferrofluid that was a mixture of $94.3 \%$ water and $5.7 \%$ cobalt ferrite nanoparticles. If we consider after the sol gel process that only $20 \%$ of the TEOS and water mixture will remain [8], it means that in the end after the solidification of the silica the cobalt ferrite particle concentration will be approximately $12.8 \%$ in the formers' matrix. This percentage of nanoparticles would be enough to make a functioning circulator [2].

\subsection{Measurement bench}

The Faraday rotation was measured on a spectral polarimeter at LT2C laboratories [9] and consists of a light source, a polarizer, electromagnets, an analyzer and a light detector figure 5 . The sample is to be placed within the electromagnet poles.

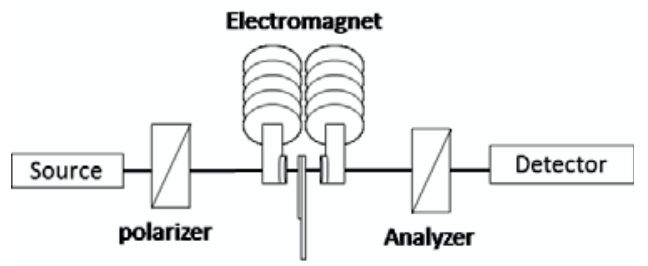

Fig. 5. Schematic of the measurement bench.

\section{Results and discussion}

After preparing the samples, the Faraday rotation cycle was measured using a spectral polarimeter in LT2C laboratory [9],to inspect the orientation of the nanoparticles. When a linearly polarized light wave passes through the sample in the presence of a magnetic field parallel to the propagation of the light, the formers' linear polarization state will convert into an elliptical one and will be characterized by two angles; the Faraday rotation $\left(\theta_{\mathrm{F}}\right)$ and the Faraday ellipticity $\left(\varepsilon_{\mathrm{F}}\right)$ [9] as can be seen in figure 6.

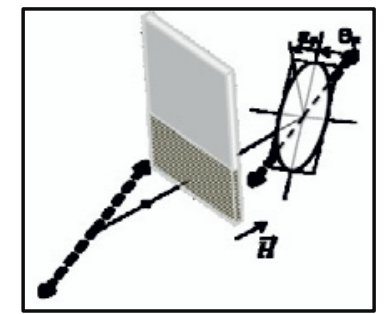

Fig. 6.Schematic representation of the Faraday effects. 
The Faraday rotation was presented as function of the applied magnetic field during the measurement at a specific wavelength chosen according to less noise factor and more signal intensity of the Faraday rotation and ellipticity cycles.

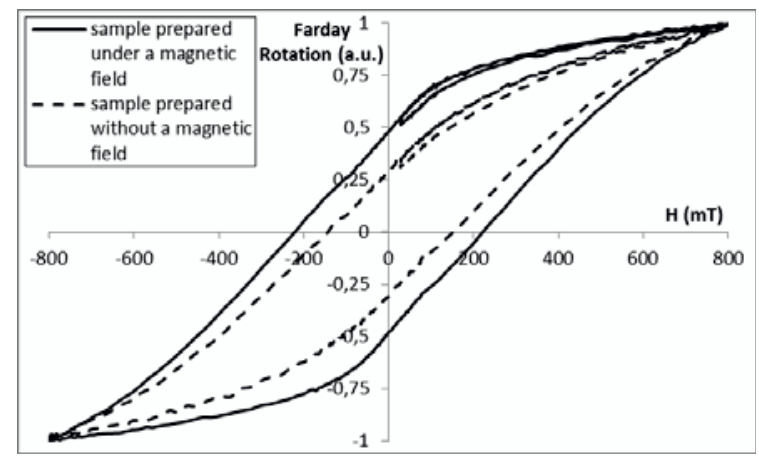

Fig. 7. Graphic comparison of Faraday rotation of a sample prepared without applying a magnetic field and a sample processed (dip coted and left to dry under a magnetic field).

For the two samplesin figure 7, there is an obvious difference in the Faraday rotation cycles thus we can see that for the sample that was prepared without a magnetic field the coercive field is approximately $150 \mathrm{mT}$ and the remanent 0.25 (normalized), on the other hand for the sample processed under a specific magnetic field the coercive an remanent field are $225 \mathrm{mT}$ and 0.5 (normalized) respectively, therefore an increase in both coercitive and remanent field by $50 \%$ and $100 \%$ respectively thus confirming the fact that the nanoparticles were and have stayed oriented during the measurement under another magnetic field and the hysteresis cycle is getting closer to that of a hard magnetic material and one can confirm the possibility of particle orientation in the silica matrix.

\section{Conclusions}

In this paper a circulator based on a composite magnetic substrate was simulated according to $\mathrm{CoFe}_{2} \mathrm{O}_{4}$ concentration. Nanoparticle orientation in a silica matrix was verified. These results are very encouraging and promising;however improvements must still be made to have a better remanent field capable of functioning properly a non-reciprocal passive microwave component, namely the coplanar circulator, at high frequencies. Nonetheless these results are enough to proceed to the next step and do a magnetophoretic(or dip coating) deposition of Cobalt ferrite nanoparticles in porous alumina membranes to create an oriented composite substrate.

\section{References}

1. M. Darques, J. De la Torre Medina, L. Piraux, L. Cagnon and I. Huynen, Nano.21,145208 (2010)

2. T. Boyajian, D. Vincent, S. Neveu, M. LeBerre, J.J. Rousseau,Micr.Symp. Digest (MTT), IEEE MTT-S International(2011)
3. A. Saib, D. Vanhoenacker-Janvier, J.-P. Raskin, A. Crahay and I. Huynen, IEEE Nano-Dev. (2001)

4. V. B. Bregar, IEEE Trans. On Magn. 40.3, 1679 (2004).

5. B.M. Hutchins, M. Platt, W.O. Hancock and M.E. Williams, Micro\& Nano Letters 1.1 , 47-52 (2006)

6. L.Ph. Carignan, Ch. Lacroix, A. Ouimet, M. Ciureanu, A. Yelon and D. Ménard, Jour. Of Appl. Phys. 102, 023905 (2007)

7. V. Boucher and D. Ménard, J. of Apl. phys. 103 07E720 (2008)

8. A.M. Buckley and M. Greenblatt, J. Chem. Ed. 71.7, 599 (1994)

9. E. Abou Diwan, F. Royer, R. Kekesi, MF. BlancMignon, S. Neveu, JJ. Rousseau,Proc. SPIE 8767, Int. Phot. : Mat., Dev., and Appl. II, 87670W (2013). 